\title{
Phage Capsid-like Structure of Myxococcus xanthus Encapsulin, a Protein Shell That Stores Iron
}

\author{
Juan Fontana ${ }^{1}$, Daniel Nemecek ${ }^{1}$, Colleen A. McHugh ${ }^{2}$, Anastasia A. Aksyuk ${ }^{1}$, Naiqian Cheng ${ }^{1}$, Dennis \\ C. Winkler ${ }^{1}$, J. Bernard Heymann $^{1}$, Egbert Hoiczyk ${ }^{2}$, and Alasdair C. Steven ${ }^{1}$ \\ 1. Laboratory of Structural Biology Research, National Institute of Arthritis and Musculoskeletal and \\ Skin Diseases, National Institutes of Health, Bethesda, MD, 20892, U.S.A. \\ 2. Department of Molecular Microbiology and Immunology, Johns Hopkins University, Baltimore, MD \\ 21205, U.S.A.
}

Iron is both an essential cofactor of many enzymes and a producer of highly reactive hydroxyl radicals that can cause cellular damage. To regulate the supply of intracellular iron, cells have developed protein-based organelles, like ferritins, that act as iron storage containers. Myxococcus xanthus, a soil-dwelling gram-negative myxobacterium, produces another type of protein-based organelle that has been related to iron metabolism, the encapsulin nanocompartment [1]. It has a spherical shell composed of a $32 \mathrm{kDa}$ protein called EncA or encapsulin, and three minor proteins in the $11 \mathrm{kDa}$ to $17 \mathrm{kDa}$ range [1]. To gain insight on the molecular architecture of this complex, we have studied encapsulin nanocompartments purified from M. xanthus and recombinant EncA shells produced in E. coli using single-particle cryo-electron microscopy (cryo-EM). This analysis was supplemented with conventional TEM with and without negative staining and scanning transmission electron microscopy (STEM).

Cryo-electron micrographs were recorded on a CM200 FEG microscope operated at $120 \mathrm{kV}$ and a magnification of X 50,000; and on a Polara operated at $300 \mathrm{kV}$ and X 59,000. The images were recorded on Kodak SO163 film and digitized with a Nikon Super Coolscan 9000ED camera. From the spherical appearance of the particles, we anticipated that they conform to icosahedral symmetry. We therefore sought to perform a three-dimensional reconstruction that exploits this symmetry focusing on the EncA shells. To initiate the iterative projection-matching procedure, random orientations were assigned to the projections. A density map using $~ 8,000$ projections from data acquired on the CM200 microscope, yielded a reconstruction at $\sim 8 \AA$ resolution (FSC 0.3 cut-off) using Bsoft [2]. To improve the resolution, we then selected $\sim 14,000$ particles from the Polara micrographs and analyzed these data using as reference the CM200 reconstruction filtered to $15 \AA$. To avoid overfitting, particles were separated into two halves and reconstructed independently. The resulting maps yielded a reconstruction at $\sim 4.6 \AA$ resolution (FSC 0.143 cut-off). This was clearly interpretable as a $\mathrm{T}=3$ (180-subunit) particle whose subunit had a HK97-like fold [3]. To carry the analysis further, we generated a model by homology modeling [4] starting from the encapsulin from Thermotoga maritima (pdb 3DKT), and subsequently performed flexible fitting [5] to improve the agreement with the EM density map. The resulting atomic coordinates were refined using crystallographic procedures [6].

M. xanthus encapsulin particles are $\sim 32 \mathrm{~nm}$ in diameter. Imaged by multiple techniques, they are seen to contain electron-dense material consistent with its being iron-rich deposits (Figure 1A-D). This core material dominates the images and appears not to be organized with high regularity. Accordingly, for a three-dimensional reconstruction, we focused our attention on the recombinant EncA particles from $E$. coli, which are of the same size but lack the dense core (Figure 1E). These data yielded a structure at 4.6 A resolution (Figure 2A). Further analyzed by homology modeling, flexible fitting, and crystallographic refinement procedures, this density map led to an atomic model of EncA (Figure 2B). It discloses a subunit fold that is closely related to that of the HK97 capsid protein (Figure 2C) although it assembles 
into a smaller shell $(\mathrm{T}=3,32 \mathrm{~nm}$ diameter, vs. $\mathrm{T}=7,45 \mathrm{~nm}$ diameter) and has an additional $\alpha$-helix at the $\mathrm{N}$-terminus. This structural similarity, which has interesting evolutionary implications, implies that essentially the same building-block can alternatively be deployed to form protective shells for viral genomes in transit between cells or as an iron-sequestering nanocompartment.

\section{References:}

[1] C.A. McHugh et al. In preparation.

[2] J.B. Heymann and D.M. Belnap. J. Struct. Biol. 157 (2007), 3-18.

[3] W.R. Wikoff et al. Science 289 (2000), 2129-2133.

[4] Y. Zhang. BMC Bioinformatics 9 (2008), 40.

[5] L.G. Trabuco et al. Structure 16 (2008), 673-683.

[6] P.V. Afonine, R.W. Grosse-Kunstleve and P.D. Adams. CCP4 Newsl. 42 (2005), contribution 8.

[7] We thank Dr. Weimin Wu for his help with single-particle reconstruction. This research was supported by the Intramural Research Program of the National Institute of Arthritis and Musculoskeletal and Skin Diseases of the National Institutes of Health.
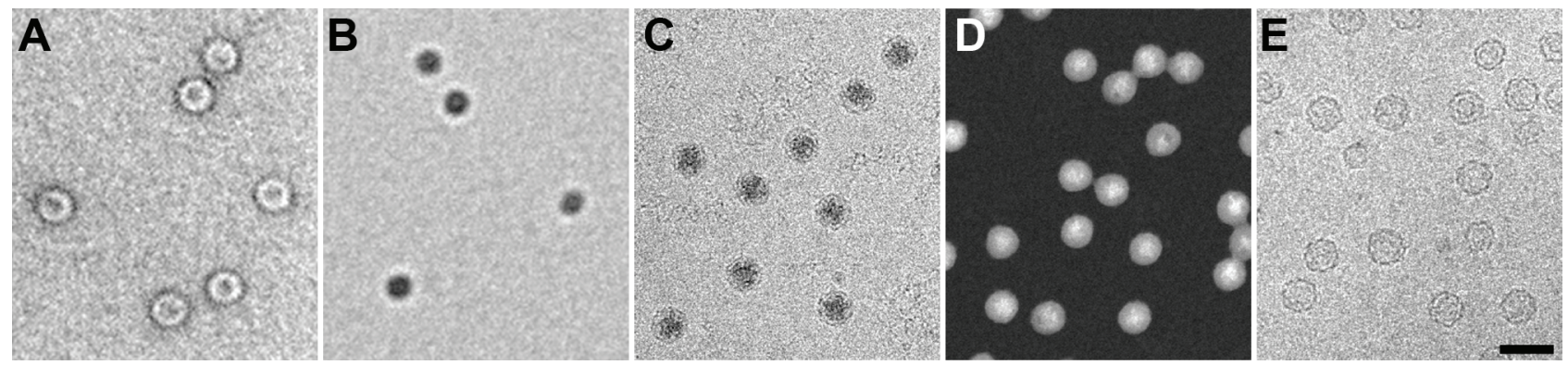

Figure 1. M. xanthus encapsulin nanocompartments imaged by: A, negative staining; B, unstained; C, cryo-EM; and D, STEM. E, E. coli-expressed EncA shells imaged by cryo-EM. Bar, 50 nm.
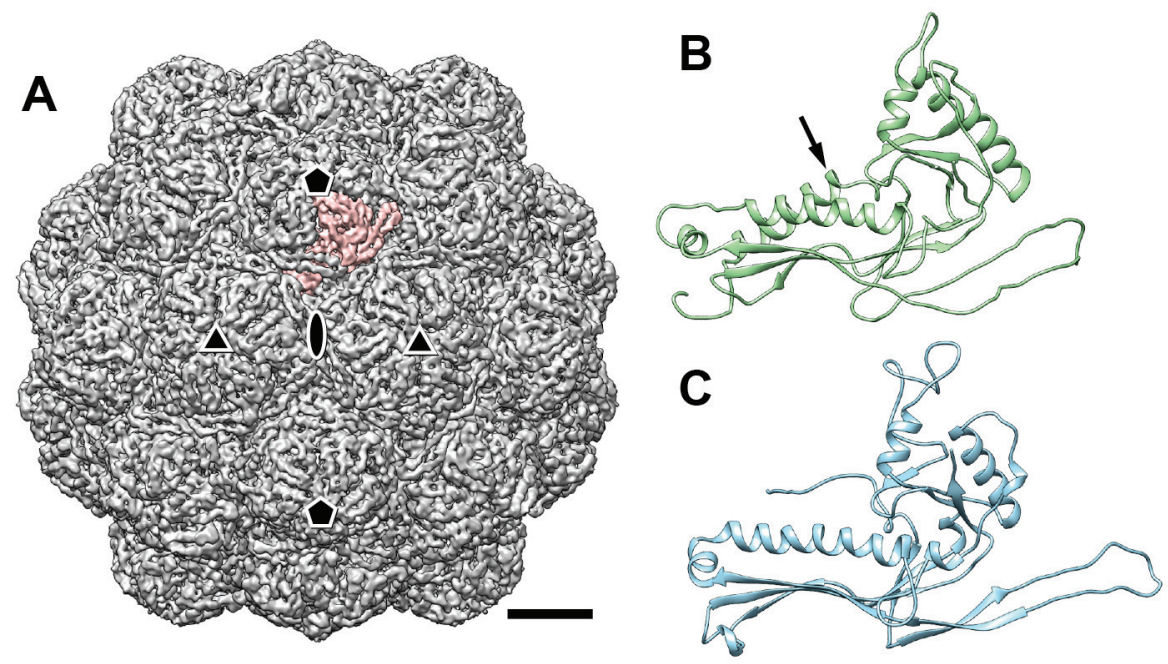

Figure 2. A, Isosurface rendering of the EncA $\mathrm{T}=3$ shell single-particle cryo-EM reconstruction. An EncA monomer is colored in pink. B, EncA atomic model derived from the EM map. The arrow marks the N-terminal $\alpha$-helix. C, HK97 gp5* in its Head II conformation (pdb 2FT1). Bar, $5 \mathrm{~nm}$. 